\title{
LOCALLY LIPSCHITZ CONTINUOUS PERTURBATIONS OF LINEAR DISSIPATIVE OPERATORS AND NONLINEAR SEMIGROUPS
}

\author{
SHINNOSUKE OHARU AND TADAYASU TAKAHASHI
}

\begin{abstract}
Locally Lipschitz continuous perturbations of linear $m$-dissipative operators in Banach spaces are considered from the point of view of the nonlinear semigroup theory. A necessary and sufficient condition is given for a semilinear operator $A+F$ to be the infinitesimal generator of a nonlinear semigroup which provides mild solutions (with exponential growth) of the semilinear evolution equation $u^{\prime}(t)=(A+F) u(t)$. It turns out that a characterization of Hille-Yosida type for nonlinearly perturbed contraction semigroups is obtained.
\end{abstract}

Introduction. This paper is concerned with the characterization of nonlinear semigroups which provide mild solutions of semilinear evolution equations in $\mathrm{Ba}$ nach spaces of the form

$$
u^{\prime}(t)=(A+F) u(t), \quad t>0 .
$$

In (DE) the operator $A$ is assumed to be the infinitesimal generator of a linear contraction semigroup $\{T(t): t \geq 0\}$ of class $\left(C_{0}\right)$ in a Banach space $X$ and $F$ a nonlinear operator from a convex subset $C$ of $X$ into $X$ which is Lipschitz continuous in a local sense.

Equation (DE) does not necessarily admit strong solutions and the variation of parameters formula

$$
u(t)=T(t) x+\int_{0}^{t} T(t-s) F u(s) d s, \quad t \geq 0,
$$

is employed to define the generalized solutions of (DE). Namely, an $X$-valued continuous function $u$ on $[0, \infty)$ satisfying $u(t) \in C$ and (IE) for $t \geq 0$ is called a mild solution on $[0, \infty)$ of (DE) with $u(0)=x \in C$. Further, the integral equation (IE) may have only local solutions provided $F$ is locally Lipschitz continuous in a proper sense. We then introduce a lower semicontinuous functional $p$ on $X$ into $[0, \infty)$ to define the local Lipschitz continuity of $F$ and specify the growth of solutions $u(\cdot)$ of (IE) in terms of the real-valued functions $p(u(\cdot))$. In case of concrete partial differential equations the use of such a functional $p$ corresponds to a priori estimates or energy estimates which ensure the global existence of the solutions.

In this paper we assume that the functional $p$ is convex, $C$ is contained in the effective domain $D(p)=\{x \in X: p(x)<\infty\}$, and that $F$ is Lipschitz continuous

Received by the editors March 24, 1986.

1980 Mathematics Subject Classification (1985 Revision). Primary 58D25, 47H20; Secondary 47H17.

Key words and phrases. Nonlinear perturbations of linear dissipative operators, semilinear evolution equations, mild solutions, nonlinear semigroups, measure of noncompactness.

The first author's research was supported by the Grant-in-Aid for Scientific Research from the Ministry of Education of Japan. 
on the level sets $C_{r}=\{x \in C: p(x) \leq r\}, r \geq 0$. Under these assumptions we think of mild solutions $u(\cdot)$ of (DE) such that

$$
p(u(t)) \leq e^{a t}(p(x)+b t) \quad \text { for } t \geq 0,
$$

where $a, b$ are nonnegative constants. Our objective here is to establish a necessary and sufficient condition for $A+F$ to be the infinitesimal generator of a nonlinear semigroup $\{S(t): t \geq 0\}$ on $C$ such that for $x \in C$ the $C$-valued continuous function $u(t) \equiv S(t) x$ on $[0, \infty)$ is a unique mild solution of (DE) satisfying the growth condition $(\mathrm{G})$. The sufficiency of the condition yields a generation theorem for nonlinear semigroups as mentioned above, while the necessity of the condition implies that the infinitesimal generator of the semigroup $\{S(t)\}$ on $C$ is densely defined in $C$ and exactly equal to the semilinear operator $A+F$. It turns out that a semilinear Hille-Yosida theorem is obtained.

The sufficiency of the condition can be proved by applying well-known results in the theory of operator semigroups. Hence most of this paper is devoted to the proof of the necessity. For this purpose we employ here the measure of noncompactness, and our method depends heavily upon the condition that $F$ is locally Lipschitz continuous in the above-mentioned sense. Semilinear equations of the form (DE) have been studied by many authors, although this paper is particularly related to the work of Martin [5] and Webb [8].

1. Main result. Let $X$ be a Banach space with norm $|\cdot|$ and $C$ a fixed convex subset of $X$. In what follows, $p$ denotes a proper lower semicontinuous functional on $X$ into $[0, \infty]$ and it is assumed without further mention that $p$ is convex, $C \subset D(p)$, and that the level set $C_{r} \equiv\{x \in C: p(x) \leq r\}$ is closed for each $r \geq 0$.

The identity operator on $X$ is denoted by $I$. Let $\{T(t): t \geq 0\}$ be a $\left(C_{0}\right)$ semigroup of linear contraction operators in $X$ and $A$ the infinitesimal generator of $\{T(t)\}$ with domain $D(A)$. It is well known (see for instance Pazy's book [7]) that $A$ is a densely defined, closed linear operator in $X$ and the resolvent $(I-\lambda A)^{-1}$ of $A$ exists as a linear contraction operator in $X$ for $\lambda>0$. Further, $F$ is a nonlinear operator from $C$ into $X$ and we assume throughout this paper that $F$ is Lipschitz continuous on $C_{r}, r>0$. Namely, for each $r>0$ there is a constant $\omega_{r}$ such that

$$
|F x-F y| \leq \omega_{r}|x-y| \quad \text { for } x, y \in C_{r} \text {. }
$$

If in particular $p(x)=|x|$ for $x \in X$, then the above condition on $F$ states that $F$ is Lipschitz continuous on bounded subsets of $C$; if $p(x) \equiv 0$ on $X$, then $C_{r} \equiv C$ and $F$ is globally Lipschitz continuous on $C$. In this paper we are concerned with the semilinear operator $A+F$ with domain $D(A) \cap C$ which is a locally Lipschitz continuous perturbation of a linear $m$-dissipative operator.

Let $x \in C$ and consider the initial-value problem for (DE):

$$
u^{\prime}(t)=(A+F) u(t), \quad t>0 ; \quad u(0)=x \in C .
$$

An $X$-valued continuous function $u(\cdot)$ on $[0, \infty)$ is said to be a mild solution of $(1.2)$ if $u(t) \in C$ for $t \geq 0, F u(\cdot)$ is continuous on $[0, \infty)$, and $u(\cdot)$ satisfies

$$
u(t)=T(t) x+\int_{0}^{t} T(t-s) F u(s) d s \quad \text { for } t \geq 0 .
$$

Moreover, a mild solution $u(\cdot)$ of (1.2) is said to be locally p-bounded if $\sup _{0 \leq t \leq \tau} p(u(t))<\infty$ for $\tau>0$. For locally $p$-bounded mild solutions we have the following uniqueness theorem. 
Proposition. Let $x, y \in C$ and let $u(\cdot), v(\cdot)$ be mild solutions of $(\mathrm{DE})$ satisfying $u(0)=x$ and $v(0)=y$, respectively. Suppose that for each $\tau>0$ there is $r(\tau)$ such that $p(u(t)), p(v(t)) \leq r(\tau)$ for $t \in[0, \tau]$. Then

$$
|u(t)-v(t)| \leq \exp \left(\omega_{r(\tau)} t\right)|x-y| \quad \text { for } t \in[0, \tau] \text { and } \tau>0 .
$$

PROOF. Applying (1.1) to (1.3), we obtain

$$
|u(t)-v(t)| \leq|x-y|+\omega_{r(\tau)} \int_{0}^{t}|u(s)-v(s)| d s \quad \text { for } t \in[0, \tau] .
$$

Hence the usual estimation of Gronwall's type implies (1.4). Q.E.D.

Before stating our main result we recall the notion of semigroup of local Lipschitzian operators on $C$. By a continuous semigroup on $C$ is meant a one-parameter family $\{S(t): t \geq 0\}$ of (possibly nonlinear) continuous operators from $C$ into itself such that $S(0) x=x, S(t+s) x=S(t) S(s) x$ for $s, t \geq 0, x \in C$, and $S(t) x$ is continuous in $t \geq 0$ for each $x \in C$. A continuous semigroup $\{S(t)\}$ on $C$ is said to be locally equi-Lipschitz continuous on $C$ if for $r>0$ and $\tau>0$ there is a nonnegative constant $\omega(r, \tau)$ such that

$$
|S(t) x-S(t) y| \leq \exp (\omega(r, \tau) t)|x-y|
$$

for $t \in[0, \tau]$ and $x, y \in C_{r}$.

THEOREM 1. Let $a, b \geq 0$. The following two conditions are equivalent:

(I) There exists a continuous semigroup $\{S(t): t \geq 0\}$ on $C$ satisfying

(I.1) $S(t) x=T(t) x+\int_{0}^{t} T(t-s) F S(s) x d s$ for $t \geq 0$ and $x \in C$,

(1.2) $p(S(t) x) \leq e^{a t}(p(x)+b t)$ for $t \geq 0$ and $x \in C$.

(II) Given $r \geq 0$ there exists a number $\lambda_{0} \equiv \lambda_{0}(r) \in(0,1 / a)$ such that to each $x \in C_{r}$ and each $\lambda \in\left(0, \lambda_{0}\right)$ there corresponds $x_{\lambda} \in D(A) \cap C$ satisfying

(II.1) $x_{\lambda}-\lambda(A+F) x_{\lambda}=x$,

(II.2) $p\left(x_{\lambda}\right) \leq(1-a \lambda)^{-1}(p(x)+b \lambda)$.

Condition (I.1) together with (I.2) means that for each $x \in C$, the function $u(t) \equiv S(t) x$ gives a locally $p$-bounded mild solution of (1.2). Hence the above Proposition states that a continuous semigroup $\{S(t)\}$ satisfying (I) is uniquely determined by the operators $A$ and $F$, and that $\{S(t)\}$ is locally equi-Lipschitz continuous on $C$. Moreover, it follows from condition (II) that $D(A) \cap C$ is dense in $C$. In fact, let $x \in C, r=p(x), \lambda_{0}$ be a number in $(0,1 / a)$ determined by (II), and let $R=\left(1-a \lambda_{0}\right)^{-1}\left(r+b \lambda_{0}\right)$. Then for each $\lambda \in\left(0, \lambda_{0}\right)$ there is $x_{\lambda} \in D(A) \cap C$ satisfying (II.1) and (II.2). Since $x, x_{\lambda} \in C_{R}, x_{\lambda}=(I-\lambda A)^{-1}\left(x+\lambda F x_{\lambda}\right)$, and $(I-\lambda A)^{-1}$ is nonexpansive, we have

$$
\begin{aligned}
\left|x_{\lambda}-x\right| & \leq \lambda\left|F x_{\lambda}-F x\right|+\left|(I-\lambda A)^{-1}(x+\lambda F x)-x\right| \\
& \leq \lambda \omega_{R}\left|x_{\lambda}-x\right|+\lambda|F x|+\left|(I-\lambda A)^{-1} x-x\right|,
\end{aligned}
$$

and so $x_{\lambda} \rightarrow x$ as $\lambda \downarrow 0$. This shows that $C$ is contained in the closure of $D(A) \cap C$. On the other hand, a semigroup $\{S(t)\}$ on $C$ satisfying (I) has the property that $\lim _{t \downarrow 0} t^{-1}(S(t) x-x)$ exists iff $x \in D(A) \cap C$, and the limit is equal to $(A+F) x$. Therefore the infinitesimal generator of $\{S(t)\}$ is exactly the semilinear operator $A+F$ with domain $D(A) \cap C$, and the implication (I) $\Rightarrow$ (II) states that the infinitesimal generator $A+F$ is densely defined. Combining the above-mentioned, we obtain the following semilinear Hille-Yosida theorem. 
THEOREM 2. The semilinear operator $A+F$ is the infinitesimal generator of a continuous semigroup $\{S(t)\}$ on $C$ satisfying (I.1) and (I.2) iff the domain $D(A) \cap C$ is dense in $C$ and for each $r>0$ there is $\lambda_{0} \in(0,1 / a)$ such that $R\left(I-\lambda\left(A+F_{R}\right)\right) \supset$ $C_{r}$ and

$$
p\left(\left(I-\lambda\left(A+F_{R}\right)\right)^{-1} x\right) \leq(1-a \lambda)^{-1}[p(x)+b \lambda] \quad \text { for } \lambda \in\left(0, \lambda_{0}\right),
$$

where $R=\left(1-a \lambda_{0}\right)^{-1}\left(r+b \lambda_{0}\right)$ and $F_{R}$ denotes the restriction of $F$ to $C_{R}$.

As will be seen in the next section, the semigroup $\{S(t)\}$ is determined through the exponential formula

$$
S(t) x=\lim _{n \rightarrow \infty}\left(I-\frac{t}{n}\left(A+F_{R}\right)\right)^{-n} x, \quad x \in C_{r}, t \in[0, \tau],
$$

where $r>0, \tau>0$, and $R>e^{a \tau}(r+b \tau)$. If in particular $p \equiv 0$ and $a=b=0$ in the above theorem, then $A+F$ is a Lipschitz perturbation of the linear $m$-dissipative operator $A$ and the necessary and sufficient condition for $A+F$ reads as follows: $A+F$ is densely defined in $C$ and satisfies the range condition $R(I-\lambda(A+F)) \supset C$ for $\lambda>0$ sufficiently small.

2. Proof of Theorem 1. We first prove the implication (II) $\Rightarrow$ (I) by applying basic results on linear and nonlinear semigroups.

Let $\lambda>0, x \in C$, and set $R=e^{2 a \tau}(p(x)+b \tau)$. Let $\lambda_{0}$ be a number $\lambda_{0}(R) \in$ $(0,1 / a)$ determined by condition (II) and choose a positive integer $n_{0}$ so that $\tau / n<$ $\lambda_{0} / 2$ for $n \geq n_{0}$. Put $h_{n}=\tau / n$ for $n \geq n_{0}$. Then it follows from (II) that for each $n \geq n_{0}$ there exists a finite sequence $\left(x_{k}^{n}\right)_{k=0}^{n}$ such that $x_{0}^{n}=x, x_{k}^{n} \in D(A) \cap C$,

$$
x_{k}^{n}-h_{n}(A+F) x_{k}^{n}=x_{k-1}^{n} \quad \text { and } \quad p\left(x_{k}^{n}\right) \leq\left(1-a h_{n}\right)^{-1}\left(p\left(x_{k-1}^{n}\right)+b h_{n}\right)
$$

for $k=1, \ldots, n$. Since

$$
p\left(x_{k}^{n}\right) \leq\left(1-a h_{n}\right)^{-k}\left(p(x)+b k h_{n}\right) \leq e^{2 a \tau}(p(x)+b \tau)=R
$$

for $k=1, \ldots, n$ and $n=1,2, \ldots$, and since the operator $A+F-\omega_{R} I$ is dissipative on $C_{R}$, the convergence theorems established in [2 and 4] can be applied to ensure that there is an $X$-valued continuous function $u(\cdot ; x)$ on $[0, \tau]$ such that $x_{k}^{n}$ converges to $u(t ; x)$ as $n \rightarrow \infty$ and $k h_{n} \rightarrow t$ in $[0, \tau]$. It is clear that $u(t ; x) \in C$ for $t \in[0, \tau]$. The first inequality in (2.2) and the lower semicontinuity of $p$ together imply that the limit function $u(\cdot ; x)$ satisfies

$$
p(u(t ; x)) \leq e^{a t}(p(x)+b t)
$$

for $t \in[0, \tau]$. On the other hand, by (2.1) and by induction we obtain

$$
x_{k}^{n}=\left(1-h_{n} A\right)^{-k} x+h_{n} \sum_{j=1}^{k}\left(I-h_{n} A\right)^{-(k-j+1)} F x_{j}^{n}
$$

for $k=1, \ldots, n$. Passing to the limit as $n \rightarrow \infty$, we can show in the same way as in $[8]$ that the limit function $u(\cdot ; x)$ satisfies

$$
u(t ; x)=T(t) x+\int_{0}^{t} T(t-s) F u(s ; x) d s
$$


for $t \in[0, \tau]$. Since $\tau>0$ is arbitrary, it is concluded that for each $x \in C$ there is a unique $X$-valued continuous function $u(\cdot ; x)$ on $[0, \infty)$ satisfying $(2.3)$ and $(2.4)$ for all $t \geq 0$. We then define a family $\{S(t): t \geq 0\}$ of operators from $C$ into itself by

$$
S(t) x=u(t ; x) \quad \text { for } t \geq 0 \text { and } x \in C .
$$

It is easy to check that $\{S(t)\}$ forms a continuous semigroup on $C$ satisfying (I.1) and (I.2), and thus (I) is obtained.

We next prove the implication (I) $\Rightarrow$ (II) via a series of lemmas. Assume in the following that (I) holds. Fix any $r \geq 0$ and set

$$
R=e^{a}(r+1+b) \quad \text { and } \quad \lambda_{0}=\min \left\{1 / 2 \omega_{R}, 1 /(a(r+1)+b)\right\}
$$

where $\omega_{R}$ is the Lipschitz constant of $F$ on $C_{R}$. Moreover, for each $h>0$, we define a bounded linear operator $A_{h}$ and a nonlinear operator $F_{h}$ in $X$ by

$$
A_{h} x=h^{-1}(T(h)-I) x \quad \text { and } \quad F_{h} x=h^{-1} \int_{0}^{h} T(h-s) F S(s) x d s \quad \text { for } x \in C .
$$

For $h>0, A_{h}$ is $m$-dissipative on $X$ and $F_{h}$ is Lipschitz continuous on $C_{r}, r>0$. More precisely, given $r>0$, we have $\left|F_{h} x-F_{h} y\right| \leq \omega_{r(h)}|x-y|$ for $x, y \in C_{r}$, where $r(h)=e^{a h}(r+b h)$ and $\omega_{r(h)}$ is defined by (1.1) with $r$ replaced by $r(h)$. We note that $\lim _{h \downarrow 0} A_{h} x=A x$ for $x \in D(A)$ and $\lim _{h \downarrow 0} F_{h} x=F x$ for $x \in C$.

LEMMA 1. Let $x \in C_{r}$. Let $\lambda \in\left(0, \lambda_{0}\right)$ and $h(\lambda)=\min \left\{1, \lambda_{0}-\lambda\right\}$. For each $h \in(0, h(\lambda)]$ there exists $x_{\lambda, h} \in C_{r+1}$ with the following properties:

(i) $x_{\lambda, h}=(\lambda+h)^{-1} h x+(\lambda+h)^{-1} \lambda S(h) x_{\lambda, h}$.

(ii) $x_{\lambda, h}=\left(I-\lambda A_{h}\right)^{-1} x+\lambda\left(I-\lambda A_{h}\right)^{-1} F_{h} x_{\lambda, h}$.

(iii) $p\left(x_{\lambda, h}\right) \leq\left(1-a_{h} \lambda\right)^{-1}\left(p(x)+b_{h} \lambda\right), a_{h}=(1-a h)^{-1} a, b_{h}=(1-a h)^{-1} b$.

(iv) The mapping $h \rightarrow x_{\lambda, h}$ is continuous and uniformly bounded on $(0, h(\lambda)]$.

Proof. Let $0<h \leq h(\lambda)$ and define a mapping $G_{h}: C_{r+1} \rightarrow X$ by

$$
G_{h} y=(\lambda+h)^{-1} h x+(\lambda+h)^{-1} \lambda S(h) y \quad \text { for } y \in C_{r+1} .
$$

Since $\lambda+h \leq \lambda_{0}$ and $e^{a h} \leq(1-a h)^{-1}$, we have $(1-a h)^{-1}[a(r+1)+b] \lambda \leq 1$ and

$$
\begin{aligned}
& (\lambda+h)^{-1}\left[h r+\lambda e^{a h}(r+1+b h)\right] \\
& \quad \leq(\lambda+h)^{-1} h\left[r+\lambda(1-a h)^{-1}(a(r+1)+b)\right]+(\lambda+h)^{-1} \lambda(r+1) \leq r+1 .
\end{aligned}
$$

Using this estimate and the convexity of $p$, we have

$$
\begin{aligned}
p\left(G_{h} y\right) & \leq(\lambda+h)^{-1}[h p(x)+\lambda p(S(h) y)] \\
& \leq(\lambda+h)^{-1}\left[h r+\lambda e^{a h}(r+1+b h)\right] \leq r+1
\end{aligned}
$$

for $y \in C_{r+1}$. This shows that $G_{h}$ maps $C_{r+1}$ into itself. On the other hand, $\omega_{R}(\lambda+h)<1$, and so $\left(1-\omega_{R} h\right)^{-1} \omega_{R}<\lambda^{-1}$. Since $\exp \left(\omega_{R} h\right) \leq\left(1-\omega_{R} h\right)^{-1}$, we have $(\lambda+h)^{-1} \lambda \exp \left(\omega_{R} h\right)<1$. Hence it follows from conditions (I.1), (I.2), and the estimate (1.4) that the Lipschitz constant of $G_{h}$ is estimated as

$$
\operatorname{Lip}\left(G_{h}\right) \leq(\lambda+h)^{-1} \lambda \exp \left(\omega_{R} h\right) \leq(\lambda+h)^{-1} \lambda\left(1-\omega_{R} h\right)^{-1}<1 .
$$

Therefore by the contracting mapping principle $G_{h}$ has a unique fixed point $x_{\lambda, h}$ in $C_{r+1}$, and $x_{\lambda, h}$ satisfies equation (i). To show (ii), we note that $S(h) x_{\lambda, h}=$ $T(h) x_{\lambda, h}+h F_{h} x_{\lambda, h}$ by (I.1). This together with relation (i) implies that 
$\left(I-\lambda A_{h}\right) x_{\lambda, h}=x+\lambda F_{h} x_{\lambda, h}$. Since $\left(I-\lambda A_{h}\right)^{-1}$ exists as a contraction operator on $X$, relation (ii) is obtained. Next, the application of (I.2) and the convexity of $p$ to relation (i) yields

$$
\begin{aligned}
p\left(x_{\lambda, h}\right) & \leq(\lambda+h)^{-1}\left[h p(x)+\lambda e^{a h}\left(p\left(x_{\lambda, h}\right)+b h\right)\right] \\
& \leq(\lambda+h)^{-1}\left[h p(x)+\lambda(1-a h)^{-1}\left(p\left(x_{\lambda, h}\right)+b h\right)\right] .
\end{aligned}
$$

Solving this inequality with respect to $p\left(x_{\lambda, h}\right)$, we obtain estimate (iii). Finally, the continuity of the mapping $h \rightarrow x_{\lambda, h}$ is deduced from (2.6) and the fact that the mapping $h \rightarrow G_{h} y$ is continuous for each $y \in C_{r+1}$. Finally, the boundedness of the set $\left\{x_{\lambda, h}: h \in(0, h(\lambda)]\right\}$ follows from the estimate given in [1]:

$$
\limsup _{h \downarrow 0}\left|x_{\lambda, h}-x\right| \leq\left(1-\omega_{R} \lambda\right)^{-1}\left[2+\exp \left(-\omega_{R}\right)\left(\lambda \omega_{R}-2\right)\right] \sup _{0 \leq t \leq 1}|S(t) x-x|
$$

Q.E.D.

As mentioned before Lemma $1, \lim _{h \downarrow 0}\left(A_{h}+F_{h}\right) y=(A+F) y$ for $y \in D(A) \cap C$ if $D(A) \cap C \neq \varnothing$. This, together with relation (ii) in Lemma 1, suggests that the elements $x_{\lambda}$ as mentioned in (II) will be obtained as the limits $x_{\lambda}=\lim _{h \downarrow 0} x_{\lambda, h}$, $\lambda \in\left(0, \lambda_{0}\right)$. To find such limits, we first show by means of the measure of noncompactness that for each $\lambda \in\left(0, \lambda_{0}\right)$ the set $\left\{x_{\lambda, h}: h \in(0, h(\lambda)]\right\}$ is relatively compact in $X$. Given a bounded subset $V$ of $X$, the ball measure of noncompactness of $V$ is defined as the infimum of all numbers $\rho>0$ such that $V$ is covered by a finite number of balls of radius $\rho$; and it is denoted by $\beta(V)$.

LEMMA $2([\mathbf{3}, \mathbf{6}])$. Let $V$ and $W$ be bounded subsets of $X$. Then we have:

(i) $\beta(V+W) \leq \beta(V)+\beta(W)$, where $V+W=\{y+z: y \in V, z \in W\}$.

(ii) $\beta(\mu W)=\mu \beta(W)$ for $\mu>0$, where $\mu W=\{\mu y: y \in W\}$.

(iii) $\beta(V)=0$ iff $V$ is relatively compact.

(iv) If $W$ is relatively compact in $X$, then $\beta(V \cup W)=\beta(V)$.

In what follows, we fix an element $x \in C_{r}$. Let $\lambda \in\left(0, \lambda_{0}\right), h(\lambda)=\min \left\{1, \lambda_{0}-\lambda\right\}$, and consider the sets

$$
V_{\lambda}=\left\{x_{\lambda, h}: h \in(0, h(\lambda)]\right\} \quad \text { and } \quad W_{\lambda}=\left\{\left(I-\lambda A_{h}\right)^{-1} F_{h} x_{\lambda, h}: h \in(0, h(\lambda)]\right\} \text {, }
$$

where $x_{\lambda, h}$ denotes the element obtained for $\lambda$ and $h$ by Lemma 1 .

Lemma 3. Let $R, \lambda_{0}$ be defined by (2.5). Then $\beta\left(W_{\lambda}\right) \leq 2 \omega_{R} \beta\left(V_{\lambda}\right)$ for $\lambda \in$ $\left(0, \lambda_{0}\right)$.

ProOF. Let $\lambda \in\left(0, \lambda_{0}\right)$. Since $V_{\lambda}$ and $W_{\lambda}$ are bounded by (2.7), both $\beta\left(V_{\lambda}\right)$ and $\beta\left(W_{\lambda}\right)$ are finite. Let $\varepsilon>0, \rho=\beta\left(V_{\lambda}\right)+\varepsilon$, and let $\delta \in(0, h(\lambda))$. We define $V_{\lambda, \delta}=\left\{x_{\lambda, h}: h \in(0, \delta]\right\}$ and $W_{\lambda, \delta}=\left\{\left(I-\lambda A_{h}\right)^{-1} F_{h} x_{\lambda, h}: h \in(0, \delta]\right\}$. Since the mappings $h \rightarrow x_{\lambda, h}$ and $h \rightarrow\left(I-\lambda A_{h}\right)^{-1} F_{h} x_{\lambda, h}$ are both continuous on the interval $[\delta, h(\lambda)]$ by Lemma 1 (iv), we see from Lemma 2(iv) that $\beta\left(V_{\lambda}\right)=\beta\left(V_{\lambda, \delta}\right)$ and $\beta\left(W_{\lambda}\right)=\beta\left(W_{\lambda, \delta}\right)$. Thus we may replace $V_{\lambda}$ and $W_{\lambda}$ in the proof by $V_{\lambda, \delta}$ and $W_{\lambda, \delta}$, respectively. For $y \in X$ we write $B_{\rho}(y)=\{z \in X:|z-y| \leq \rho\}$. Then there exist a finite number of elements, say $y_{1}, \ldots, y_{n}$, of $X$ such that $V_{\lambda, \delta} \subset \bigcup_{i=1}^{n} B_{\rho}\left(y_{i}\right)$. For each $i$ choose an element $x_{\lambda, h(i)}$ satisfying $\left|x_{\lambda, h(i)}-y_{i}\right| \leq \rho$. Then $V_{\lambda, \delta} \subset$ $\bigcup_{i=1}^{n} B_{2 \rho}\left(x_{\lambda, h(i)}\right)$. Set $W_{\lambda, i}=\left\{\left(I-\lambda A_{h}\right)^{-1} F_{h} x_{\lambda, h(i)}: h \in(0, \delta]\right\}$ for $i=1, \ldots, n$. Then $W_{\lambda, i}$ are all relatively compact in $X$ since $\left(I-\lambda A_{h}\right)^{-1} F_{h} y \rightarrow(I-\lambda A)^{-1} F y$ 
as $h \downarrow 0$ for $y \in C$. Hence for each $i$ there exist a finite number of elements $z_{i, j}$, $j=1, \ldots, N(i)$, in $W_{\lambda, i}$ such that $W_{\lambda, i} \subset \bigcup_{j=1}^{N(i)} B_{\varepsilon}\left(z_{i, j}\right)$. Let $\eta=2 \omega_{R} \rho \exp \left(\omega_{R} \delta\right)+$ $\varepsilon$. Then we have the inclusion

$$
W_{\lambda, \delta} \subset \bigcup_{i=1}^{n} \bigcup_{j=1}^{N(i)} B_{\eta}\left(z_{i, j}\right)
$$

In fact, given $h \in(0, \delta], x_{\lambda, h} \in B_{2 \rho}\left(x_{\lambda, h(i)}\right)$ for some $i \in\{1, \ldots, n\}$ and there is $z_{i, j} \in W_{\lambda, i}$ such that $\left(I-\lambda A_{h}\right)^{-1} F_{h} x_{\lambda, h(i)} \in B_{\varepsilon}\left(z_{i, j}\right)$. Hence we have

$$
\begin{aligned}
\left|\left(I-\lambda A_{h}\right)^{-1} F_{h} x_{\lambda, h}-z_{i, j}\right| \leq & \left|\left(I-\lambda A_{h}\right)^{-1}\left(F_{h} x_{\lambda, h}-F_{h} x_{\lambda, h(i)}\right)\right| \\
& +\left|\left(I-\lambda A_{h}\right)^{-1} F_{h} x_{\lambda, h(i)}-z_{i, j}\right| \\
\leq & \omega_{R} \exp \left(\omega_{R} h\right)\left|x_{\lambda, h}-x_{\lambda, h(i)}\right|+\varepsilon \leq \eta,
\end{aligned}
$$

and (2.8) follows. The inclusion (2.8) yields

$$
\beta\left(W_{\lambda}\right) \leq \eta=2 \omega_{R} \exp \left(\omega_{R} \delta\right)\left(\beta\left(V_{\lambda}\right)+\varepsilon\right)+\varepsilon .
$$

Since $\varepsilon>0$ and $\delta>0$ are arbitrary, we obtain the desired assertion. Q.E.D.

LeMma 4. Let $\lambda_{0}$ be defined by (2.5). Then $\beta\left(V_{\lambda}\right)=0$ for $\lambda \in\left(0, \lambda_{0}\right)$.

Proof. Let $\lambda \in\left(0, \lambda_{0}\right)$. Then the set $K \equiv\left\{\left(I-\lambda A_{h}\right)^{-1} x: h \in(0, h(\lambda)]\right\}$ is relatively compact. Hence we infer from Lemma 1 (ii) and Lemmas 2 and 3 that

$$
\beta\left(V_{\lambda}\right) \leq \beta\left(K+\lambda W_{\lambda}\right)=\lambda \beta\left(W_{\lambda}\right) \leq 2 \lambda \omega_{R} \beta\left(V_{\lambda}\right) .
$$

Thus $\beta\left(V_{\lambda}\right)=0$. Q.E.D.

Finally, Lemma 5 below completes the proof of the implication (I) $\Rightarrow$ (II).

LEMMA 5. For $\lambda \in\left(0, \lambda_{0}\right), x_{\lambda} \equiv \lim _{h \downarrow 0} x_{\lambda, h}$ exists in $D(A) \cap C$ and satisfies

$$
x_{\lambda}-\lambda(A+F) x_{\lambda}=x \quad \text { and } \quad p\left(x_{\lambda}\right) \leq(1-a \lambda)^{-1}(p(x)+b \lambda) .
$$

Proof. Let $\lambda \in\left(0, \lambda_{0}\right)$. By Lemmas 2 and 4 there is a null sequence $(h(n))$ in $(0, h(\lambda)]$ such that $x_{\lambda, h(n)}$ converges as $n \rightarrow \infty$ to some $x_{\lambda}$. Since $x_{\lambda, h} \in C_{r+1}$ for $h \in(0, h(\lambda)], x_{\lambda} \in C_{r+1}$. Further, it follows from Lemma 1 (ii) that $x_{\lambda}$ satisfies the equation $x_{\lambda}=(I-\lambda A)^{-1}\left(x+\lambda F x_{\lambda}\right)$. Therefore $x_{\lambda}$ belongs to $D(A) \cap C$ and satisfies the first relation of (2.9). On the other hand,

$$
p\left(x_{\lambda}\right) \leq \liminf _{n \rightarrow \infty} p\left(x_{\lambda, h(n)}\right) \leq(1-a \lambda)^{-1}(p(x)+b \lambda)
$$

by Lemma 1(iii). Thus it suffices to show that there is at most one element $x_{\lambda}$ in $C_{r+1}$ satisfying (2.9). Suppose that an element $y \in C_{r+1}$ satisfies (2.9). Then $x_{\lambda}-y=\lambda(I-\lambda A)^{-1}\left(F x_{\lambda}-F y\right)$ and $\left|x_{\lambda}-y\right| \leq \lambda \omega_{r+1}\left|x_{\lambda}-y\right|$, and so $y=x_{\lambda}$. Consequently, $x_{\lambda, h}$ converges to $x_{\lambda}$ as $h \downarrow 0$. Q.E.D.

\section{REFERENCES}

1. J. Baillon, Générateurs et semi-groupes dans les espaces de Banach uniformement lisses, J. Funct. Anal. 29 (1978), 199-213.

2. M. Crandall and T. Liggett, Generation of semigroups of nonlinear transformations on general Banach spaces, Amer. J. Math. 93 (1971), 265-298.

3. K. Deimling, Ordinary differential equations in Banach spaces, Lecture Notes in Math., vol. 596, Springer-Verlag, Berlin and New York, 1977. 
4. Y. Kobayashi, Difference approximation of Cauchy problems for quasidissipative operators and generation of nonlinear semigroups, J. Math. Soc. Japan 27 (1975), 640-665.

5. R. Martin, Jr., Invariant sets for perturbed semigroups of linear operators, Ann. Math. Pura Appl. 150 (1975), 221-239.

6. __ Nonlinear operators and differential equations in Banach spaces, Wiley-Interscience, New York, 1976.

7. A. Pazy, Semignoups of linear operators and applications to partial differential equations, Appl. Math. Sci., vol. 44, Springer-Verlag, New York, 1983.

8. G. Webb, Continuous nonlinear perturbations of linear accretive operators in Banach spaces, J. Funct. Anal. 10 (1972), 191-203.

Department of MATHEMATICS, HiRoshima UNiVERSity, HiRoshima 730, JAPAN

National Aerospace Laboratory, Chofu, TOKyo, Japan 